\title{
Local vs. global hyperconvexity, tautness or $k$-completeness for unbounded open sets in $\mathbb{C}^{n}$
}

\author{
Nikolai Nikolov and Peter Pflug
}

\begin{abstract}
Some known localization results for hyperconvexity, tautness or $k$ completeness of bounded domains in $\mathbb{C}^{n}$ are extended to unbounded open sets in $\mathbb{C}^{n}$.
\end{abstract}

Mathematics Subject Classification (2000): 32A19 (primary); 32F45, 32Q45 (secondary).

\section{Introduction}

For bounded domains in $\mathbb{C}^{n}$ the following localization results are well known:

A bounded domain $D \in \mathbb{C}^{n}$ is taut, hyperconvex or Kobayashi complete if and only if $D$ is locally taut, locally hyperconvex or locally Kobayashi complete, respectively.

The aim of this note is to extend these results to unbounded domains imposing the correct conditions at infinity. So we get:

1. An unbounded open set $D \subset \mathbb{C}^{n}$, locally hyperconvex at every finite boundary point, for which $\infty$ is a plurisubharmonic (psh) peak point, is hyperconvex (cf. Proposition 2.2).

2. Let $D \subset \mathbb{C}^{n}$ be an unbounded open set for which $\infty$ is a barrier point. Then $D$ is locally taut if and only if $D$ is taut (cf. Corollary 3.5).

3. An unbounded open set $D \subset \mathbb{C}^{n}$, for which $\infty$ is a $k^{\prime}$-point, is Kobayashi complete if and only if it is locally Kobayashi complete (cf. Proposition 3.6).

Moreover, a characterization for unbounded Kobayashi hyperbolic domains is given, namely:

This paper was written during the stay of the first named author at the University of Oldenburg supported by a grant from the DAAD (November-December 2004). He would like to thank both institutions for their support.

The authors deeply thank the referee for all his remarks which improved this paper essentially. Pervenuto alla Redazione il 21 dicembre 2004 e in forma definitiva il 18 ottobre 2005. 
4. An unbounded open set $D \subset \mathbb{C}^{n}$ is Kobayashi hyperbolic if and only if for all $b \in D$ one has $\liminf _{z \rightarrow b, w \rightarrow \infty} l_{D}(z, w)>0$, where $l_{D}$ denotes the Lempert function of $D$ (cf. Proposition 3.1).

We apply the above results to characterize Hartogs domains.

The above localization results are presented for any open, not necessarily connected, subset of $\mathbb{C}^{n}$. To this end we will introduce several pointwise conditions like that of $t$-point, $k^{\prime}$-point and $k$-point.

\section{Barrier points and hyperconvex open sets}

A boundary point $a$ of an open set $D$ in $\mathbb{C}^{n}$ is said to be a (global) barrier point for $D$ if there is a negative psh function $\varphi$ on $D$ with $\lim _{z \rightarrow a} \varphi(z)=0$ (here and below $\infty \in \partial D$ if $D$ is unbounded); $\varphi$ is called a (global) barrier function for $D$ at $a$.

We call $a$ a local barrier point for $D$ if there is an open neighborhood $U$ of $a$ (neighborhood in this paper always means an open neighborhood) such that $a$ is a barrier point for $D \cap U$.

Proposition 2.1. If $\infty$ is a barrier point of an (unbounded) open set $D$ in $\mathbb{C}^{n}$, then $D$ admits a bounded strictly psh barrier function at $\infty$. In particular, any connected component of D is hyperbolic (i.e. the Kobayashi pseudodistance is a distance) [15] and possesses a Bergman metric [5]. ${ }^{1}$

Proof. Let $\psi$ be a barrier function for $D$ at $\infty$. We may assume that $-1<\psi<0$. Let $\left\{D_{j}\right\}$ be a sequence of open sets such that $D_{j} \subset \subset D_{j+1}$ and $\cup_{j=1}^{\infty} D_{j}=D$. Then there are balls $\mathbb{B}\left(0, r_{j}\right),{ }^{2} j \in \mathbb{N}$, with

$$
\alpha_{j}:=\inf _{D \backslash \mathbb{B}\left(0, r_{j}\right)} \psi>\beta_{j}:=\sup _{D_{j}} \psi .
$$

Setting

$$
\varphi_{j}(z):=\left\{\begin{array}{ll}
\psi(z), & z \in D \backslash \mathbb{B}\left(0, r_{j}\right) \\
\max \left\{\psi(z), r_{j}^{-2}\left(\alpha_{j}-\beta_{j}\right)\|z\|^{2}+\beta_{j}\right\}, & z \in D \cap \mathbb{B}\left(0, r_{j}\right)
\end{array},\right.
$$

it is easy to check that the function $\varphi:=\sum_{j=1}^{\infty} \frac{\varphi_{j}}{2^{j}}$ has the required properties.

Remark. We do not know whether the hyperbolicity of $D$ already follows by the weaker assumption that $\infty$ is a local barrier point.

On the other hand, we shall see later (Remark (ii) after Example 4.3) that there are unbounded open sets for which $\infty$ is a local barrier point but not a global one.

${ }^{1}$ The result in [5] requires the bounded strictly psh function to be continuous. However, the proof there works without this assumption.

${ }^{2} \mathbb{B}(a, r):=\mathbb{B}_{n}(a, r):=\left\{z \in \mathbb{C}^{n}:\|z-a\|<r\right\}$. 
We say that an open subset $D \subset \mathbb{C}^{n}$ is hyperconvex if there is a negative psh exhaustion function $\varphi$ of $D$, i.e. $\lim _{z \rightarrow \partial D} \varphi(z)=0$.

In the literature sometimes the definition of hyperconvexity requires the exhaustion function $\varphi$ to be continuous. But it is well known (see [19]) that both definitions are equivalent (see also Remark (ii) after the proof of Lemma 2.3).

Note that $D$ is hyperconvex if and only if any of its connected components is hyperconvex. Indeed, it suffices to consider the case when $D$ has infinitely many connected components $D_{1}, D_{2}, \ldots$ Assume that every $D_{j}$ is hyperconvex with respect to the negative psh exhaustion function $\varphi_{j}$. Then replace $\varphi_{j}, j \in \mathbb{N}$, by $\tilde{\varphi}_{j}:=\max \left\{\varphi_{j},-j^{-1}\right\}$. Hence, $D$ is hyperconvex with respect to the psh function $\tilde{\varphi}$ on $D$, where $\left.\tilde{\varphi}\right|_{D_{j}}:=\tilde{\varphi}_{j}$.

Denote by $g_{D}(a, \cdot)$ the pluricomplex Green function of $D$ with pole at $a \in D$, i.e.

$$
g_{D}(a, \cdot):=\sup \{u(\cdot)\}
$$

where the supremum is taken over all $\operatorname{psh} u: D \mapsto[-\infty, 0]$ such that $u(\cdot)-\log \|\cdot-a\|$ is bounded from above near $a$.

It is clear that if $a$ and $z$ belong to the same connected component $\tilde{D}$ of $D$, then $g_{D}(a, z)=g_{\tilde{D}}(a, z)<0$. Otherwise, $g_{D}(a, z)=0$. Recall that $g_{D}(a, \cdot)$ is a psh function.

Moreover, if $D$ is a bounded domain in $\mathbb{C}^{n}$ and $a \in D$, then it is well-know (cf. [11]) that

$$
D \text { is hyperconvex } \quad \text { if and only if } \lim _{z \rightarrow b} g_{D}(a, z)=0, b \in \partial D .
$$

We mention that from the proof of Proposition 2.2 below it will follow that the same result remains true for an unbounded open set.

Recall that a boundary point $a$ of an open set $D$ in $\mathbb{C}^{n}$ is said to be a psh peak point for $D$ if there exists a psh function $\varphi$ on $D$ with $\lim _{z \rightarrow a} \varphi(z)=0$ and $\limsup _{z \rightarrow b} \varphi(z)<0$ for any $b \in \partial D \backslash\{a\} ; \varphi$ is called a psh peak function for $D$ at $a$. Observe the local character of that notion. Moreover, any psh peak point is a barrier point.

In [9] it is shown that any bounded locally hyperconvex domain is hyperconvex. Now we extend this result to the case of unbounded open sets.

Proposition 2.2. Let $D$ be an unbounded open set in $\mathbb{C}^{n}$ which is locally hyperconvex at any finite boundary point (i.e. for any finite $a \in \partial D$ there is a neighborhood $U$ of a such that any connected component of $D \cap U$ is hyperconvex). If $\infty$ is a psh peak point, then $D$ is hyperconvex.

To prove this proposition, we shall use the following

Lemma 2.3. Let $D_{2} \subset D_{1} \subset D$ be open sets in $\mathbb{C}^{n}$ with $D_{1} \neq D$ and $\partial D_{2} \cap D \subset$ $D_{1}$. Let $\psi$ be a negative psh function on $D$ such that

$$
\alpha:=\inf _{D \cap \partial D_{1}} \psi>\beta:=\sup _{D \cap \partial D_{2}} \psi .
$$


For $a \in D_{2}, \operatorname{set} d(a):=\inf _{D \cap \partial D_{2}} g_{D_{1}}(a, \cdot)$. Then

$$
g_{D}(a, z) \geq g_{D_{1}}(a, z)+\frac{\alpha}{\beta-\alpha} d(a) \text { if } z \in D_{1},
$$

and

$$
g_{D}(a, z) \geq \frac{\psi(z)}{\beta-\alpha} d(a) \text { if } z \in D \backslash D_{1}
$$

Proof. We may assume that $d(a)>-\infty$. Then the function $u(a, z):=\frac{\psi(z)-\alpha}{\beta-\alpha} d(a)$, $z \in D$, satisfies

$$
u(a, z) \leq g_{D_{1}}(a, z), z \in D \cap \partial D_{2},
$$

and

$$
u(a, z) \geq 0 \geq \limsup _{D_{1} \ni \zeta \rightarrow z} g_{D_{1}}(a, \zeta), z \in D \cap \partial D_{1} .
$$

Therefore, it follows that

$$
v(a, z):= \begin{cases}g_{D_{1}}(a, z), & z \in D_{2} \\ \max \left\{g_{D_{1}}(a, z), u(a, z)\right\}, & z \in D_{1} \backslash D_{2} \\ u(a, z), & z \in D \backslash D_{1}\end{cases}
$$

is a psh function in the second variable with a logarithmic pole at $a$. Moreover,

$$
v(a, z) \leq \frac{\alpha}{\alpha-\beta} d(a), z \in D
$$

Hence, by definition of $g_{D}$, one has

$$
g_{D}(a, z) \geq v(a, z)+\frac{\alpha}{\beta-\alpha} d(a),
$$

which implies the desired results.

Remarks. (i) The first inequality in Lemma 2.3 is sharp. To see this, it is enough to consider a bounded hyperconvex domain $D$ and $D_{1}=\left\{w \in D: g_{D}(a, w)<\alpha\right\}$, $D_{2}=\left\{w \in D: g_{D}(a, w)<\beta\right\}$, where $a \in D$ and $\beta<\alpha<0$ (cf. [20]).

(ii) It is well known that for a bounded hyperconvex domain $D \subset \mathbb{C}^{n}$ the pluricomplex Green function $g_{D}$ is continuous on $D \times \bar{D}$, where $\left.g_{D}\right|_{D \times \partial D}:=0$ (see [11]). The same result remains true for an arbitrary hyperconvex open set $D$. In particular, $e^{g_{D}(a, \cdot)}-1, a \in D$, is a negative continuous psh exhaustion function of $D$.

Indeed, to see this, note that the second inequality in Lemma 2.3 applied for a negative psh exhaustion function of $D$ implies that $\lim _{z \rightarrow a, w \rightarrow \partial D} g_{D}(z, w)=0^{3}$ for

${ }^{3}$ Observe that we may choose $D_{2}:=\mathbb{B}(a, r) \subset \subset D_{1} \subset \subset D$ with $D_{1}$ hyperconvex. 
any $a \in D$ (this lemma also shows that $g_{D}(z, w)>-\infty$ for $z \neq w$ ). Moreover, $g_{D}$ is upper semicontinuous as a function of both variables (see [10]). Assume now that $g_{D}$ is not continuous at some point $(a, b) \in D \times D$. Then there is $\varepsilon>0$ and a sequence $\left(z_{j}, w_{j}\right) \rightarrow(a, b)$ such that $g_{D}\left(z_{j}, w_{j}\right) \leq g_{D}(a, b)-2 \varepsilon$. Let $\varphi$ be a negative psh exhaustion function of $D$. We may choose open sets $D_{2} \subset \subset D_{1} \subset \subset$ $D$ such that $D_{2} \times D_{1}$ contains the above sequence, $D_{1}$ is pseudoconvex with smooth boundary,

$$
-1 \leq \inf \left\{g_{D}\left(z_{j}, w\right): j \in \mathbb{N}, w \in D \cap \partial D_{2}\right\} \leq d\left(z_{j}\right),
$$

and

$$
\inf _{D \cap \partial D_{1}} \varphi \geq \frac{\varepsilon}{1+\varepsilon} \sup _{D \cap \partial D_{2}} \varphi .
$$

Then Lemma 2.3 implies that $g_{D}\left(z_{j}, w_{j}\right) \geq g_{D_{1}}\left(z_{j}, w_{j}\right)-\varepsilon$. The assumptions for $D_{1}$ imply that it is hyperconvex [9]. Hence $g_{D_{1}}$ is a continuous function and letting $j \rightarrow \infty$ this gives the contradiction

$$
g_{D}(a, b)-2 \varepsilon \geq g_{D_{1}}(a, b)-\varepsilon \geq g_{D}(a, b)-\varepsilon .
$$

Proof of Proposition 2.2. It is enough to show that

$$
\lim _{D \ni w \rightarrow a} g_{D}(z, w)=0
$$

for any $a \in \partial D$ and any $z \in D$.

First, let $a=\infty$. We shall prove (2.1) under the weaker condition that $\infty$ is a barrier point. By Proposition 2.1, there exists a strictly psh barrier function $\varphi$ at $\infty$. Choose a smooth function $\chi$ such that $\chi=1$ near $z$ and supp $\chi \subset \subset D$. Then there is a constant $c>0$ for which

$$
u_{z}(w):=c \varphi(w)+\chi(w) \log \|w-z\|, \quad w \in D,
$$

is a psh function on $D$ with a logarithmic pole at $z$. Hence, $g_{D}(z, w) \geq u_{z}(w)$, $w \in D$, which implies (2.1).

Now let $a \in \partial D \cap \mathbb{C}^{n}$. Let $r>0$ be such that $a, z \in \mathbb{B}(0, r)$. If $\psi$ is a psh peak function for $D$ at $\infty$, then $\sup _{D \cap \partial \mathbb{B}(0, r)} \psi<0$. In virtue of the properties of $\psi$ there is $r^{\prime}>r$ such that

$$
2 \inf _{D \cap \partial \mathbb{B}\left(0, r^{\prime}\right)} \psi>\sup _{D \cap \partial \mathbb{B}(0, r)} \psi
$$

Put $\hat{D}:=D \cap \mathbb{B}\left(0, r^{\prime}\right)$. Then, applying Lemma 2.3, we have

$$
g_{D}(z, w) \geq g_{\hat{D}}(z, w)+\inf _{D \cap \partial \mathbb{B}(0, r)} g_{\hat{D}}(z, \cdot) \geq g_{\hat{D}}(z, w)+\inf _{D \cap \partial \mathbb{B}(0, r)} g_{D}(z, \cdot) .
$$


Denote by $\tilde{D}$ the connected component of $\hat{D}$ containing $z$. Since $\tilde{D}$ is a hyperconvex domain (see [9]), it follows that $\lim _{\tilde{D} \ni w \rightarrow a} g_{\hat{D}}(z, w)=0$. On the other hand, $g_{\hat{D}}(z, w)=0$ if $w \notin \tilde{D}$. Therefore,

$$
\liminf _{w \rightarrow a} g_{D}(z, w) \geq \inf _{D \cap \partial \mathbb{B}(0, r)} g_{D}(z, \cdot)
$$

Using that $r$ was arbitrary and applying the first case, we are lead to

$$
\inf _{D \cap \partial \mathbb{B}(0, r)} g_{D}(z, \cdot) \underset{r \rightarrow \infty}{\longrightarrow} 0
$$

hence, we get (2.1).

Remarks. (i) We do not know whether Proposition 2.2 remains true if $\infty$ is a (global or local) barrier point.

On the other hand, if $\Pi$ is the upper-half plane in $\mathbb{C}$, then the unbounded domain $D:=\Pi \times \mathbb{C}$ is locally hyperconvex at any finite boundary point but $D$ is not hyperconvex.

(ii) Any hyperconvex domain $D$ admits a bounded strictly psh exhaustion function. In particular, its Bergman metric is complete [4].

Indeed, note that Proposition 2.1 implies that there is a strictly psh function $\psi$ on $D$ with $-2<\psi<-1$. Now let $\varphi$ be a psh exhaustion function with $-1<\varphi<$ 0 . Choose a sequence $\left\{D_{j}\right\}$ of domains such that $D_{j} \subset \subset D_{j+1}, \cup_{j=1}^{\infty} D_{j}=D$, and $\max _{\overline{D_{j}}} \frac{\varphi}{2} \leq b_{j}:=\inf _{D \backslash D_{j+1}} \varphi$. Then

$$
\varphi_{j}:= \begin{cases}-b_{j} \psi & \text { on } D_{j} \\ \max \left\{-b_{j} \psi, \varphi\right\} & \text { on } D_{j+1} \backslash D_{j} \\ \varphi & \text { on } D \backslash D_{j+1}\end{cases}
$$

is a psh function on $D$ with $-1<\varphi_{j}<0$. Then it is easy to check that $\tilde{\varphi}:=\sum_{j=1}^{\infty} \frac{\varphi_{j}}{2^{j}}$ is a strictly psh exhaustion function of $D$ with $-1<\tilde{\varphi}<0$.

(iii) The argument at the beginning of the proof of Proposition 2.2 implies that an open set $D$ in $\mathbb{C}^{n}$ is hyperconvex if and only if any of its boundary points is a barrier point.

More generally, Z. Blocki (see [2], Problem 1) asks whether a bounded domain in $\mathbb{C}^{n}$ is hyperconvex if any of its boundary points is a local barrier point (here the word "any" is essential - Example 4.1 in Paragraph 4 provides a bounded pseudoconvex domain for which the set of all boundary points that are not local barrier points, is a circle). In the next paragraph we shall see that such a domain is, at least, taut. 


\section{Hyperbolic, taut and $k$-complete open sets}

Recall that an open set $D$ in $\mathbb{C}^{n}$ is said to be taut if the family $\mathcal{O}(\mathbb{D}, D)$ of all holomorphic mapping from the unit disc $\mathbb{D}:=\mathbb{B}_{1}(0,1) \subset \mathbb{C}$ into $D$ is normal, i.e. any sequence $\left\{f_{j}\right\} \subset \mathcal{O}(\mathbb{D}, D)$ contains a subsequence $\left\{f_{j_{k}}\right\}$ with $f_{j_{k}} \underset{k \rightarrow \infty}{\longrightarrow}$ $f \in \mathcal{O}(\mathbb{D}, D)$ (convergence in the compact-open topology) or a subsequence that diverges compactly. Observe that this condition is equivalent to the following one: either $\left\{f_{j}\right\}$ is compactly divergent or it allows a subsequence that converges compactly uniformly to an $f \in \mathcal{O}(\mathbb{D}, D)$. It is well-known that any bounded hyperconvex domain is taut and that any taut domain is pseudoconvex and hyperbolic (cf. [11]). Note that the unbounded hyperconvex domains are also taut (cf. [14] or combine Propositions 2.1 and 3.4 below).

We say that $a \in \partial D$ is a locally taut point for $D$ if there is a neighborhood $U$ of $a$ such that the set $D \cap U$ is taut. $D$ is said to be locally taut if any finite boundary point of $D$ is a locally taut point for $D$. Obviously, any boundary point of a taut open set is a locally taut point.

Note that $D$ is taut if and only if any of its connected components is taut. Indeed, it is enough to show that if $D$ has infinitely many connected components $D_{1}, D_{2}, \ldots$, and $\left\{f_{j}\right\} \subset \mathcal{O}(\mathbb{D}, D)$ such that $\#\left\{j: f_{j}(\mathbb{D}) \subset D_{k}\right\}<\infty, k \in \mathbb{N}$, then $\left\{f_{j}\right\}$ diverges compactly. To see this, it suffices to observe that any $K \subset \subset$ $D$ intersects finitely many connected components of $D$ by Lebesgue's covering theorem.

For a domain $D$ in $\mathbb{C}^{n}$, denote by $l_{D}$ its Lempert function, i.e.

$$
l_{D}(z, w):=\inf \{|\lambda|: \exists \varphi \in \mathcal{O}(\mathbb{D}, D) \text { with } \varphi(0)=z \text { and } \varphi(\lambda)=w\} .
$$

Let now $D$ be an open set in $\mathbb{C}^{n}$, and $z, w \in D$. If $z, w$ belong to the same connected component $\tilde{D}$ of $D$, then we set $l_{D}(z, w):=l_{\tilde{D}}(z, w)$. Otherwise, we define $l_{D}(z, w):=1$.

We call $a \in \partial D$ a (global) $t$-point for $D$ if

$$
\lim _{z \rightarrow a, w \rightarrow b} l_{D}(z, w)=1
$$

for any $b \in D$. Note that

$a$ is a t-point if and only if for any $\left\{f_{j}\right\} \subset \mathcal{O}(\mathbb{D}, D)$ with $f_{j}(0) \rightarrow a$

it follows that $\left\{f_{j}\right\}$ diverges compactly.

We say that $a \in \partial D$ is a local $t$-point for $D$ if there is a neighborhood $U$ of $a$ such that $a$ is a $t$-point for $D \cap U$. Thus, any locally taut point is a local $t$-point.

On the other hand, there are $t$-points which are not locally taut points. For example, for small $\varepsilon>0$ the domain

$$
D_{\varepsilon}:=\left\{(z, w) \in \mathbb{C}^{2}:|z+1|^{2}+|w|^{2}|\sin | w^{-1}||<1,|z|<2,|w|<\varepsilon\right\}
$$


is non-pseudoconvex at $(0,0)$ but $\left|e^{z}\right|-1$ is a barrier function for $D_{\varepsilon}$ at the origin and so $(0,0)$ is a $t$-point of $D_{\varepsilon}$ (cf. Proposition 3.4 below).

For convenience, we say that an open set $D \subset \mathbb{C}^{n}$ is hyperbolic if any of its connected components is hyperbolic.

That tautness implies hyperbolicity is also reflected by the following result.

Proposition 3.1. Any connected component of an unbounded open set $D$ in $\mathbb{C}^{n}$ is hyperbolic if and only if

$$
\liminf _{z \rightarrow \infty, w \rightarrow b} l_{D}(z, w)>0
$$

for any $b \in D$.

Proof. Recall that $D$ is hyperbolic if and only if the family $\mathcal{O}(\mathbb{D}, D)$ is evenly continuous (cf. [1], [11]), i.e. for any $b \in D$ and any neighborhood $U$ of $b$ there are a neighborhood $V$ of $b$ and $s \in(0,1)$ such that if $f(0) \in V$, then $f(s \mathbb{D}) \subset U$ for any $f \in \mathcal{O}(\mathbb{D}, D)$.

Using this criterion it is clear that, if $D$ is hyperbolic, then (3.1) holds.

To prove the converse assume that $D$ is not hyperbolic. Then (in virtue of the cited criterion) we may find a point $b \in D$ and sequences $\left\{s_{j}\right\} \subset \mathbb{D},\left\{f_{j}\right\} \subset$ $\mathcal{O}(\mathbb{D}, D)$ with $s_{j} \rightarrow 0, f_{j}(0) \rightarrow b, f_{j}\left(s_{j}\right) \not \rightarrow b$. If $0<r:=\liminf _{z \rightarrow \infty, w \rightarrow b} l_{D}(z, w)$, then $f_{j}(0) \rightarrow b$ implies that $\left\{f_{j}\right\}$ is locally bounded on $r \mathbb{D}$ and therefore $f_{j}\left(s_{j}\right) \rightarrow$ $b$ by Montel's theorem giving a contradiction.

Proposition 3.2. Let $D$ be an open set in $\mathbb{C}^{n}$. Assume that $\infty$ is a $t$-point if $D$ is unbounded. Then the following conditions are equivalent:

(i) D is taut;

(ii) any finite boundary point of $D$ is a locally taut point;

(iii) any finite boundary point of $D$ is a local t-point;

(iv) any finite boundary point of $D$ is a t-point.

Proof. It is clear that (iv) $\leftrightarrow$ (i) $\rightarrow$ (ii) $\rightarrow$ (iii). Thus, it suffices to show that (iii) $\rightarrow$ (i). Consider an arbitrary sequence $\left\{f_{j}\right\} \subset \mathcal{O}(\mathbb{D}, D)$. Assume that it is not compactly divergent. Since $\infty$ is a $t$-point, it easily follows that this sequence has a subsequence which is locally bounded. Thus, we may assume that $f_{j} \rightarrow f \in$ $\mathcal{O}(\mathbb{D}, \bar{D})$. It suffices to show that if $a:=f(0) \in \partial D$, then $f(\mathbb{D}) \subset \partial D$. Let $U$ be a neighborhood of $a$ such that $a$ is a $t$-point of $D \cap U$. Observe that there is $r \in(0,1]$ with $f_{j}(r \mathbb{D}) \subset U$ for any $j$ large enough. Then, by $(*), f(r \mathbb{D}) \subset \partial D$. Let now $r_{0} \in(0,1]$ be the biggest one with the last property, i.e. $r_{0}:=\sup \{r \in$ $(0,1]: f(r \mathbb{D}) \subset \partial D\}$. Assuming $r_{0}<1$, it follows that $f\left(\overline{r_{0} \mathbb{D}}\right) \subset \partial D$. Using that for any $s$ with $|s|=r_{0}, f(s)$ is a local $t$-point and applying a compactness argument, we conclude (as above) that there is $r_{1} \in\left(r_{0}, 1\right]$ with $f\left(\overline{r_{1} \mathbb{D}}\right) \subset \partial D$, giving a contradiction. 
Remarks. (i) The assumption in Proposition 3.2 that $\infty$ is a global $t$-point cannot be replaced by only assuming $\infty$ to be a local $t$-point. Indeed, $\infty$ and 0 are local $t$-point of $\mathbb{C}^{*}$ but $\mathbb{C}^{*}$ is not even hyperbolic.

(ii) Example 4.1 in Paragraph 4 will show that the set of all non-local $t$-points of a bounded pseudoconvex domain may be very small, e.g. a circle.

(iii) In [18], Theorem 2.5, the following statement may be found:

An unbounded domain $D \subset \mathbb{C}^{n}$ which is locally taut is taut if and only if $D$ has the Schottky property.

There the Schottky property is defined as follows (see [18], Definition 2.3): $D$ has the Schottky property for $h_{\mathbb{C}^{n}}$ (here $h_{\mathbb{C}^{n}}$ is the Euclidean metric of $\mathbb{C}^{n}$ ) if for every point $p \in D$, each relatively compact open set $W \subset \mathbb{C}^{n}$, and any $r \in(0,1)$ there exists $S>0$ such that every $f \in \mathcal{O}(\mathbb{D}, D), f(0) \in W$, satisfies $\|p-f(\lambda)\| \leq$ $S, \lambda \in r \overline{\mathbb{D}}$.

The following example may show that with this definition of the Schottky property the above characterization is not true. Namely, take

$$
D:=\left\{(z, w) \in \mathbb{C}^{2}: 0<|w| e^{\varphi(z)}<1\right\}
$$

where $\varphi$ is a subharmonic function on $\mathbb{C}$, bounded from below, with $\varphi(z) \underset{|z| \rightarrow \infty}{\longrightarrow} \infty$ and $\varphi(z)=\varphi(|z|), z \in \mathbb{C}$. The $D$ does not fulfill the above Schottky property. Indeed, take $p:=\left(0, w_{0}\right) \in D$ and $W:=\mathbb{B}\left(\left(0, w_{0}\right),\left|w_{0}\right|\right) \subset \subset \mathbb{C}^{2}$, and $f_{R, \delta}(\lambda):=$ $(R \lambda, \delta), \lambda \in \mathbb{D}$.

On the other side, using Hurwitz's and Montel's theorems it is easy to verify that $D$ is nevertheless taut.

With the correct definition of the Schottky property, i.e. requiring that the open set $W$ in the above definition is relatively compact in $D$, the equivalence of (i) and (ii) in Proposition 3.2 is essentially contained in [18]. not taut.

We do not know any example of a hyperbolic domain which is locally taut but

In [3] a non trivial example of an unbounded hyperbolic domain is discussed. This domain is also locally taut. As an application of Proposition 3.2 we now show that it is taut.

Let $X$ be any closed, 1-dimensional complex subvariety of $\mathbb{C}^{2}$. In [3] (see also [7]), a domain $\Omega \subset \mathbb{C}^{2}$ containing $X$ with $\bar{\Omega} \neq \mathbb{C}^{2}$ and a biholomorphic map $\Phi$ from $\Omega$ onto $\mathbb{C}^{2}$ are constructed such that $D:=\mathbb{C}^{2} \backslash \Phi(X)$ is a hyperbolic domain. Using the ideas from [3] and Proposition 3.2, unfortunately it turns out that one has

Example 3.3. The domain $D$ from [3] is taut.

Proof. Since $\Phi(X)$ is an analytic subset of $\mathbb{C}^{2}$, it follows from Montel's and Hurwitz's theorems that any point of $\Phi(X)$ is a local $t$-point of $D$. 
To see that $\infty$ is a $t$-point for $D$, we use that $\Phi$ is the limit of biholomorphic maps $\Phi_{l}: \mathbb{C}^{2} \rightarrow \mathbb{C}^{2}, l \in \mathbb{N}$, which have the following property:

$$
\text { if } \varphi \in \mathcal{O}(\mathbb{D}, \mathbb{B}(0, l+2) \backslash \Phi(X)), \varphi(0) \in \mathbb{B}(0, l / 2)
$$

and $\operatorname{dist}\left(\varphi(0), \Phi_{l}(X)\right) \geq 1 / l$, then $\varphi\left(\left(1-2^{-l}\right) \mathbb{D}\right) \subset \mathbb{B}(0, l+1)$.

Let now $\left\{\varphi_{j}\right\} \subset \mathcal{O}(\mathbb{D}, D)$ be such that $\varphi_{j}(0) \rightarrow b \in D$ as $j \rightarrow \infty$. Fixing $r \in$ $(0,1)$ we may find $m \in \mathbb{N}$ with $\prod_{k=m}^{\infty}\left(1-2^{-k}\right) \geq r$ and $\operatorname{dist}\left(\varphi_{j}(0), \Phi_{l}(X)\right) \geq 1 / l$ for any $j, l \geq m$. For given $j \geq m$, let $l \geq m$ be such that $\varphi_{j}(r \mathbb{D}) \subset \mathbb{B}(0, l+2)$. Then $\varphi_{j}\left(r\left(1-2^{-l}\right) \mathbb{D}\right) \subset \mathbb{B}(0, l+1)$ and, by induction, $\varphi_{j}(r s \mathbb{D}) \subset \mathbb{B}(0, m+1)$, where $s:=\prod_{k=m}^{l}\left(1-2^{-k}\right)$. Hence $\varphi_{j}\left(r^{2} \mathbb{D}\right) \subset \mathbb{B}(0, m+1)$ for any $j \geq m$ and thus

$$
\liminf _{z \rightarrow \infty, w \rightarrow b} l_{D}(z, w) \geq r^{2} .
$$

Letting $r \rightarrow 1$ shows that $\infty$ is a $t$-point for $D$ and, therefore, $D$ is taut.

In connection with the above example, it would be interesting to know whether there is a domain $\mathbb{C}^{2} \backslash X$ with $X$ an analytic subset of $\mathbb{C}^{2}$ such that $D$ is hyperbolic but not taut.

On the other hand, there is the following sufficient criterion for a $t$-point.

Proposition 3.4. Any barrier point of a hyperbolic open set $D$ in $\mathbb{C}^{n}$ is a t-point.

Proof. Let $a \in \partial D$ and let $\varphi$ be a barrier function at $a$. Assume that $a$ is not a $t$-point. Then there exist $\left\{f_{j}\right\} \subset \mathcal{O}(\mathbb{D}, D)$ and $\left\{t_{j}\right\} \subset \mathbb{D}$ with $f_{j}(0) \rightarrow a$, $t_{j} \rightarrow t_{0} \in \mathbb{D}$, and $f_{j}\left(t_{j}\right) \rightarrow b \in D$. Since $D$ is hyperbolic, it follows that for any neighborhood $V$ of $b$ there is a neighborhood $U$ of $t_{0}$ such that $f_{j}(U) \subset V$ for any large $j$. We may assume that $V \subset \subset D, U \subset r \mathbb{D}$ for some $r \in(0,1)$, and $f_{j}(U) \subset V$ for any $j$. Then we find $\varepsilon<0$ with $\int_{r \mathbb{D}} \varphi \circ f_{j}<\varepsilon$ for any $j$. On the other hand, the last integral is larger or equal $\pi r^{2} \varphi\left(f_{j}(0)\right)$. Using $\varphi\left(f_{j}(0)\right) \rightarrow 0$, we get a contradiction.

Combining Propositions 3.2 and 3.4 leads to the following result.

Corollary 3.5. If any finite boundary point of an unbounded open set $D$ in $\mathbb{C}^{n}$ is a locally taut point and $\infty$ is a barrier point, then $D$ is taut.

In particular, if any finite boundary point is a local barrier point and $\infty$ is a barrier point, then $D$ is taut.

Remark. The first part of Corollary 3.5 is contained as Proposition 2 in [8] (see also Corollary 2.10 in [18]).

Recall now that the Kobayashi pseudodistance $k_{D}$ of a domain $D$ in $\mathbb{C}^{n}$ is the largest pseudodistance which does not exceed $\tanh ^{-1} l_{D}$. The Kobayashi pseudometric $\kappa_{D}$ at a point $(z, X) \in D \times \mathbb{C}^{n}$ is defined as

$$
\kappa_{D}(z ; X):=\inf \left\{\alpha>0: \exists \varphi \in \mathcal{O}(\mathbb{D}, D) \text { with } \varphi(0)=z \text { and } \alpha \varphi^{\prime}(0)=X\right\} .
$$


There is a tight relationship between the Kobayashi pseudodistance and metric, namely (cf. [11]),

$$
k_{D}(z, w)=\inf \left\{\int_{0}^{1} \kappa_{D}\left(\gamma(t) ; \gamma^{\prime}(t)\right) d t\right\},
$$

where the infimum is taken over all piecewise $C^{1}$-curves $\gamma:[0,1] \rightarrow D$ connecting $z$ and $w$.

It is well-know that a bounded domain $D$ in $\mathbb{C}^{n}$ is $k$-complete (i.e. complete w.r.t. to the Kobayashi pseudodistance) if and only if $D$ is locally $k$-complete (cf. [6], [11]).

Similarly to the Lempert function, we may define the Kobayashi pseudodistance for an open set $D$ in $\mathbb{C}^{n}$. If $z, w$ belong to the same connected component $\tilde{D}$ of $D$, then we set $k_{D}(z, w):=k_{\tilde{D}}(z, w)$. Otherwise, we put $k_{D}(z, w):=\infty$.

Then it is clear that $D$ is $k$-complete if and only if any of its connected component is $k$-complete.

Now we introduce the notions of (local) $k$-points and $k^{\prime}$-points for an open set $D \subset \mathbb{C}^{n}$.

We say that $a \in \partial D$ is a $k$-point for $D$ if

$$
\lim _{z \rightarrow a} k_{D}(z, b)=\infty
$$

for any $b \in D$ (equivalently, for one $b \in D$ from any connected component of $D$ ). For example, it is essentially shown in [8] (see also [12]) that any local holomorphic peak point (possible $\infty$ ) is a $k$-point.

We call $a \in \partial D$ a $k^{\prime}$-point for $D$ if there is no $k_{D}$-Cauchy sequence converging to $a$. It is clear that any $k$-point is a $k^{\prime}$-point. The notions of local k-points and local $k^{\prime}$-points may be defined in an obvious way. Then we have

Proposition 3.6. Let $D$ be an open set in $\mathbb{C}^{n}$. Assume that $\infty$ is a $k^{\prime}$-point if $D$ is unbounded. The following conditions are equivalent:

(i) D is k-complete;

(ii) any finite boundary point of $D$ admits a neighborhood $U$ such that $D \cap U$ is k-complete;

(iii) any finite boundary point of $D$ is a local $k^{\prime}$-point;

(iv) any finite boundary point of $D$ is a $k^{\prime}$-point;

(v) any boundary point of $D$ is a local k-point;

(vi) any boundary point of $D$ is a k-point.

Proof. It is trivial that (i) $\rightarrow$ (ii) $\rightarrow$ (iii), and (vi) $\rightarrow$ (v) $\rightarrow$ (iii). We also have (i) $\rightarrow$ (vi) (cf. Theorem 7.3.2 in [11]).

Observe that the assumption that $\infty$ is a $k^{\prime}$-point implies

$$
\liminf _{w \rightarrow \infty} k_{D}(z, w)>0, \quad z \in D
$$


(if this is not the case, use the triangle inequality to get a contradiction). Hence, $D$ is hyperbolic by Proposition 4 and thus any $k_{D}$-Cauchy sequence has at most one accumulation point in $D$. Since any boundary point is a $k^{\prime}$-point, it follows that such a sequence has no accumulation points on $\partial D$. So (iv) $\rightarrow$ (i).

Therefore, it is enough to show that (iii) $\rightarrow$ (iv).

Assume the contrary. Then there exists a $k_{D}$-Cauchy sequence $\left\{z_{j}\right\} \subset D$, $z_{j} \rightarrow a \in \partial D \backslash\{\infty\}$.

Claim. There is $r>0$ such that

$$
3 \varepsilon:=\inf \left\{k_{D}\left(z_{j}, w\right): j \in \mathbb{N}, w \in D \backslash \mathbb{B}(0, r)\right\}>0 .
$$

Otherwise, for any $l$ there are $j_{l} \in \mathbb{N}$ and $w_{l} \in D$ with $\left\|w_{l}\right\|>l$ and $k_{D}\left(z_{j_{l}}, w_{l}\right)<\frac{1}{l}$. Then

$$
k_{D}\left(w_{l}, w_{m}\right)<\frac{1}{l}+\frac{1}{m}+k_{D}\left(z_{j_{l}}, z_{j_{m}}\right), \quad l, m \in \mathbb{N} .
$$

If the sequence $\left\{j_{l}\right\}$ consists of only a finite number of elements of $\mathbb{N}$, then, by considering a subsequence if necessary, we may assume that $j_{l}=j_{m}$, for any $l$ and $m$ and, hence, $\left\{w_{l}\right\}$ is a $k_{D}$-Cauchy sequence. Otherwise, we may assume that $j_{l} \rightarrow \infty$. Then $\left\{w_{l}\right\}$ is again a $k_{D}$-Cauchy sequence, since $z_{j_{l}}$ is one. This is a contradiction, since $\infty$ is a $k^{\prime}$-point. So the claim is proved.

Now we may assume that $k_{D}\left(z_{l}, z_{m}\right)<\varepsilon$ for any $l, m$ and consider a piecewise $C^{1}$-curve $\gamma_{l, m}:[0,1] \rightarrow D$ connecting $z_{l}$ and $z_{m}$ such that

$$
k_{D}\left(z_{l}, z_{m}\right) \leq \int_{0}^{1} \kappa_{D}\left(\gamma_{l, m}(t) ; \gamma_{l, m}^{\prime}(t)\right) d t<2 k_{D}\left(z_{l}, z_{m}\right)<2 \varepsilon .
$$

Since $k_{D}\left(z_{l}, \gamma_{l, m}(t)\right)<2 \varepsilon$, it follows that $\gamma_{l, m}([0,1]) \subset \tilde{D}:=D \cap \mathbb{B}(0, r)$ and

$$
\tanh ^{-1} l_{D}\left(\gamma_{l, m}(t), w\right) \geq k_{D}\left(\gamma_{l, m}(t), w\right)>\varepsilon, t \in[0,1], w \in D \backslash \mathbb{B}(0, r) .
$$

Then the inequality (cf. the proof of Proposition 7.2.9 in [11])

$$
\kappa_{\tilde{D}}(z ; X) \inf _{w \in D \backslash \mathbb{B}(0, r)} l_{D}(z, w) \leq \kappa_{D}(z ; X), z \in \tilde{D},
$$

implies that

$$
k_{\tilde{D}}\left(z_{l}, z_{m}\right)<2(\operatorname{coth} \varepsilon) k_{D}\left(z_{l}, z_{m}\right) .
$$

Hence $\left\{z_{j}\right\}$ is a $k_{\tilde{D}^{-}}$-Cauchy sequence.

Now choose $r^{\prime}>0$ such that $a$ is a $k^{\prime}$-point for $\hat{D}:=D \cap \mathbb{B}\left(a, r^{\prime}\right)$. Since $\tilde{D}$ is bounded one has

$$
\inf \left\{k_{\tilde{D}}\left(z_{j}, w\right): j \gg 1, w \in \tilde{D} \backslash \mathbb{B}\left(a, r^{\prime}\right)\right\}>0
$$

and then repeating the argument of the previous paragraph, we see that the $k_{\tilde{D}^{-}}$ Cauchy sequence $\left\{z_{j}\right\}$ is also a $k_{\hat{D}}$-Cauchy sequence, giving a contradiction. So (iii) implies (iv). 
Remark. This proof shows that any local $k^{\prime}$-point of a bounded domain is a global one. The same is not true for $k$-points. Indeed, let $\Pi$ be the upper half-plane in $\mathbb{C}$, $H:=\Pi \times \mathbb{C}, \mathbb{B}_{2}:=\mathbb{B}_{2}(0,1)$, and $D:=2 \mathbb{B}_{2} \backslash \overline{\mathbb{B}_{2} \cap H}$. Note that 0 is a $k$-point for $D \cap \mathbb{B}_{2}$, since this domain is convex, but it is not even a $t$-point for $D$.

Note that if $\infty$ is a local peak point one has

Proposition 3.7. If $\infty$ is a psh peak point and a local $k^{\prime}$-point of an unbounded open set $D$ in $\mathbb{C}^{n}$, then $\infty$ is a $k^{\prime}$-point.

Proof. Let $r>0$ be such that such that $\infty$ is a $k^{\prime}$-point for $\tilde{D}:=D \backslash \overline{\mathbb{B}(0, r)}$. Then one has

$$
\kappa_{\tilde{D}}(z ; X) \inf _{w \in D \cap \overline{\mathbb{B}(0, r)}} l_{D}(z, w) \leq \kappa_{D}(z ; X), z \in \tilde{D},
$$

On the other hand, if $r_{2}>r_{1}>r$, then

$$
\begin{aligned}
& \inf \left\{\tanh ^{-1} l_{D}(z, w): z \in D \backslash \mathbb{B}\left(0, r_{1}\right), w \in D \cap \overline{\mathbb{B}(0, r)}\right\} \\
& \geq \inf \left\{k_{D}(z, w): z \in D \cap \partial \mathbb{B}\left(0, r_{1}\right), w \in D \cap \partial \mathbb{B}(0, r)\right\} \\
& \geq \min \left\{k_{D \cup \mathbb{B}\left(0, r_{2}\right)}(z, w): z \in \partial \mathbb{B}\left(0, r_{1}\right), w \in \partial \mathbb{B}(0, r)\right\}=: \varepsilon .
\end{aligned}
$$

Since the notion of psh peak point is of a local character, one has that $\infty$ is a psh peak point for $D \cup \mathbb{B}\left(0, r_{2}\right)$. Thus, by Proposition 1 , any connected component of this set is hyperbolic and hence $\varepsilon>0$. So

$$
\kappa_{\tilde{D}}(z ; X) \leq(\operatorname{coth} \varepsilon) \kappa_{D}(z ; X), z \in D \backslash \mathbb{B}\left(0, r_{1}\right) .
$$

Assume now that there exists a $k_{D}$-Cauchy sequence converging to $\infty$. Repeating the argument of the proof of Proposition 3.6, it follows that all the members of this sequence belonging to $\tilde{D}$ form a $k_{\tilde{D}}$-Cauchy sequence, which is a contradiction.

\section{Hartogs domains}

Let $G$ be a domain in $\mathbb{C}^{n}$. Recall that a domain $D \subset G \times \mathbb{C}^{m}$ is called a Hartogs domain over $G$ with m-dimensional (balanced) fibers if

$$
D=\left\{(z, w) \in G \times \mathbb{C}^{m}: h(z, w)<1\right\}
$$

where $h: G \times \mathbb{C}^{m} \rightarrow[0, \infty)$ is an upper semicontinuous function with $h(z, \lambda w)=$ $|\lambda| h(z, w), z \in G, \lambda \in \mathbb{D}, w \in \mathbb{C}^{m}$ (cf. [12]). It clear that $D$ is bounded if and only if $G$ is bounded and $\inf _{G \times \mathbb{S}} h>0$, where $\mathbb{S}$ is the unit sphere in $\mathbb{C}^{m}$. Note also that $D$ is pseudoconvex if and only if $G$ is pseudoconvex and $\log h$ is psh.

In the following example, which is inspired by [9], different kinds of boundary points appear. 
If

$$
\begin{aligned}
& \theta\left(z_{1}\right):=\sum_{j=1}^{\infty} 2^{-j} \log \left|1-2^{2 j-1} z_{1}\right|, \\
& \varphi\left(z_{1}\right):=\max \left\{\left|z_{1}\right|, 1+\theta\left(z_{1}\right)\right\}
\end{aligned}
$$

then

$$
D:=\left\{\left(z_{1}, z_{2}\right) \in \mathbb{D} \times \mathbb{C}:\left|z_{2}\right| e^{\varphi\left(z_{1}\right)}<1\right\}
$$

is a bounded pseudoconvex Hartogs domain over $\mathbb{D}$ with one-dimensional fibers. Let $E_{0}$ be the set of all boundary points $\left(a_{1}, a_{2}\right)$ of $D$ with $a_{1} \neq 0$. Set

$$
\begin{aligned}
& E_{1}:=\left\{\left(0, a_{2}\right) \in \mathbb{C}^{2}:\left|a_{2}\right|=1\right\}, \\
& E_{2}:=\left\{\left(0, a_{2}\right) \in \mathbb{C}^{2}: e^{-1}<\left|a_{2}\right|<1\right\}, \\
& E_{3}:=\left\{\left(0, a_{2}\right) \in \mathbb{C}^{2}:\left|a_{2}\right|=e^{-1}\right\} .
\end{aligned}
$$

Observe that $\partial D=\cup_{j=1}^{4} E_{j}$. One has

\section{Example 4.1.}

(i) Any point of $E_{0} \cup E_{1}$ is a barrier point and, therefore, by Proposition 3.4, a $t$-point;

(ii) any point of $E_{2}$ is a local barrier point but not a global $t$-point and hence, by Proposition 3.4, a local $t$-point which is not a global barrier point; in particular, by Propostion 3.2, $D$ is not a taut domain;

(iii) any point of $E_{3}$ is not a local $t$-point and thus not a local barrier point.

In particular, $\partial D \backslash E_{3}$ is the set of local $t$-points. Then, applying Proposition 3.2 for a neighborhood of any of these points, it follows that $\partial D \backslash E_{3}$ is also the set of all locally taut points.

Proof. (i) Note that $\varphi$ is a continuous function on $\mathbb{C}^{*}$ Hence the points of $E_{0}$ are barrier points. Moreover, $0>\log \left|z_{2}\right| \rightarrow 0$ as $D \ni\left(z_{1}, z_{2}\right) \rightarrow E_{1}$. Thus the points of $E_{1}$ are also barrier points.

(ii) Let $a:=\left(0, a_{2}\right) \in E_{2}$. We may assume that $a_{2}>0$. Choose $c \in\left(a_{2}, 1\right)$ and observe that the discs $f_{j}(t):=\left(2^{1-2 j}, c t\right)$ belong to $\mathcal{O}(\mathbb{D}, D)$ if $2^{1-2 j} \leq$ $-\log c$. Since $f_{j}(t) \rightarrow f(t):=(0, c t)$ locally uniformly, $f(0) \in D$ and $f\left(a_{2} / c\right)=$ $a$, it follows that $a$ is not a $t$-point.

To see that $a$ is a local barrier point, observe first that if $F$ is the union of disjoint discs $\mathbb{D}\left(2^{1-2 j}, 2^{-2 j}\right), j=1,2, \ldots$, then

$$
\lim _{F \not \nexists z_{1} \rightarrow 0} \theta\left(z_{1}\right)=0 .
$$


Indeed, $\log \left|1-2^{2 j-1} z_{1}\right| \geq-\log 2$ for any $z_{1} \notin F$ and any $j \geq 1$, hence

$$
\theta\left(z_{1}\right) \geq \sum_{j=1}^{k} 2^{-j} \log \left|1-2^{2 j-1} z_{1}\right|-\log 2 \sum_{j=k+1}^{\infty} 2^{-j}, k \geq 1
$$

Letting $F \not \supset z_{1} \rightarrow 0, k \rightarrow \infty$, and recalling that $\theta$ is upper semicontinuous, one obtains the desired equality.

Note now that if $C$ is the complement of the closed disc $r \overline{\mathbb{D}}$ with $e^{-1}<r<1$ and $z \in D \cap(\mathbb{D} \times C)$, then

$$
\theta\left(z_{1}\right)<-\log (r e)<0
$$

From above it follows that there exists $\varepsilon>0$ such that if $z_{1}$ satisfies this inequality and $\left|z_{1}\right|<\varepsilon$, then $z_{1} \in F$. Hence

$$
D \cap(\varepsilon \mathbb{D} \times C) \subset F \times C .
$$

Defining $p(z):=-j^{-1}$ on $\mathbb{D}\left(2^{1-2 j}, 2^{-2 j}\right) \times C$, it follows that $p$ is a barrier function for $D \cap(\varepsilon \mathbb{D} \times C)$ at any $a$ with $a_{1}=0$ and $r<\left|a_{2}\right|<1$. Since $r \in\left(e^{-1}, 1\right)$ was arbitrary chosen, we are done.

(iii) It follows from (i), (ii) and Proposition 3.2 that the set of boundary points which are not local $t$-points is non-empty and it is contained in $E_{3}$. Since $E_{3}$ is an orbit w.r.t. $z_{2}$-rotations, this implies the statement.

Remark. It is also possible to prove (iii) directly by constructing suitable discs as in the proof of (ii).

In [17] (see also [12]) it is shown that if $D$ is hyperbolic, then

$$
G \text { is hyperbolic and } \inf _{K \times \mathbb{S}} h>0 \text { for any } K \subset \subset G \text {. }
$$

Indeed, since $G \times\{0\} \subset D$, then $G$ is hyperbolic. Assume now that $\inf _{K \times \mathbb{S}} h=0$ for some $K \subset \subset G$. Then there is a sequence $D \supset\left\{a_{j}\right\}$ and a point $a:=\left(a^{\prime}, a^{\prime \prime}\right) \in$ $G \times \mathbb{C}^{m}, a^{\prime \prime} \neq 0$, such that $a_{j} \rightarrow a$ and $h\left(a_{j}\right) \rightarrow 0$ as $j \rightarrow \infty$. Note that the $\operatorname{discs} f_{j}(t):=\left(a_{j}^{\prime}, t a_{j}^{\prime \prime}\left(h\left(a_{j}\right)\right)^{-1}\right)$ belong to $\mathcal{O}(\mathbb{D}, D), f_{j}(0) \rightarrow \tilde{a}:=\left(a^{\prime}, 0\right) \in D$, and $f_{j}(t) \rightarrow \infty$ for any $t \in \mathbb{D} \backslash\{0\}$. Hence $\liminf _{z \rightarrow \infty, w \rightarrow \tilde{a}} l_{D}(z, w)=0$, which contradicts Proposition 4.

For the special case of Hartogs domains over $\mathbb{D}$ with 1-dimensional fibers the converse to $(* *)$ is shown in [17]. In [12], the converse in general is posed as an open question. Here we obtain a positive answer as a consequence of Proposition 3.1 .

Proposition 4.2. If (**) holds, then $D$ is hyperbolic. 
Proof. Assume the contrary. It follows from Proposition 3.1 that we may find sequences $\left\{t_{j}\right\} \subset \mathbb{D}$ and $\left\{f_{j}\right\} \subset \mathcal{O}(\mathbb{D}, D)$ with $t_{j} \rightarrow 0, f_{j}\left(t_{j}\right) \rightarrow \infty$ and $f_{j}(0) \rightarrow a \in D$. Let $a=\left(a^{\prime}, a^{\prime \prime}\right)$ with $a^{\prime} \in G$ and $a^{\prime \prime} \in \mathbb{C}^{m}$. Then $f_{j}^{\prime}\left(t_{j}\right) \rightarrow a^{\prime}$ by the hyperbolicity of $G$. Hence $f_{j}^{\prime}\left(t_{j}\right) \subset K$ for some $K \subset \subset D$. Then $f_{j}^{\prime \prime}\left(t_{j}\right) \rightarrow \infty$ and one has

$$
1>h\left(f\left(t_{j}\right)\right)=\left\|f^{\prime \prime}\left(t_{j}\right)\right\| h\left(f^{\prime}\left(t_{j}\right), \frac{f^{\prime \prime}\left(t_{j}\right)}{\left\|f^{\prime \prime}\left(t_{j}\right)\right\|}\right) \geq\left\|f^{\prime \prime}\left(t_{j}\right)\right\| \inf _{K \times \mathbb{S}} h \rightarrow \infty,
$$

which is a contradiction.

The next example shows that the limit in Proposition 3.1 can be positive but less than 1.

Example 4.3. There is a hyperbolic unbounded domain in $\mathbb{C}^{3}$ for which $\infty$ is not a $t$-point.

Proof. Note that the function

$$
\Phi\left(z_{1}, z_{2}\right):= \begin{cases}\max \left\{\left|z_{1}\right|, 1-\left|e z_{2}\right|\right\}, & \left|z_{2}\right|<e^{-1} \\ \left|z_{1}\right|, & \left|z_{2}\right| \geq e^{-1}\end{cases}
$$

is positive and continuous on the domain $D$ from Example 4.1 above. Hence, by Proposition 4.2,

$$
\tilde{D}:=\{(z, w) \in D \times \mathbb{C}:|w| \Phi(z)<1\}
$$

is a hyperbolic Hartogs domain.

Now fix $c \in\left(e^{-1}, 1\right)$. Observe that the disc $f_{j}(t):=\left(2^{1-2 j}, c t\right)$ belongs to $\mathcal{O}(\mathbb{D}, D)$ for any $j$ large enough. If $g_{j}(t):=(\text { cet })^{j}$, then $\left|g_{j}(t)\right|<1 \leq$ $\min \left\{2^{2 j-1},(1-c e|t|)^{-1}\right\}$ when $c e|t|<1$, and $\left|g_{j}(t)\right|<2^{2 j-1}$ for $t \in \mathbb{D}$ and $j \geq 2$, i.e. $\left|g_{j}(t)\right| \Phi\left(f_{j}(t)\right)<1$ on $\mathbb{D}$. Hence $h_{j}:=\left(f_{j}, g_{j}\right) \in \mathcal{O}(\mathbb{D}, \tilde{D})$ with $h_{j}(0) \rightarrow 0 \in \tilde{D}$. Moreover, for any $t$ with $c e|t|>1$, we have $h_{j}(t) \rightarrow \infty$. Thus

$$
\liminf _{u \rightarrow \infty, v \rightarrow 0} l_{\tilde{D}}(u, v) \leq(c e)^{-1} .
$$

For $c \rightarrow 1$ one has

$$
\liminf _{u \rightarrow \infty, v \rightarrow 0} l_{\tilde{D}}(u, v) \leq e^{-1} .
$$

Thus $\infty$ is not a $t$-point for $D$.

Remarks. (i) In fact, the last limit is equal to $e^{-1}$. Indeed, if $\tilde{D} \supset\left(\left(z^{j}, w^{j}\right)\right)_{j} \rightarrow$ $\infty$, then it is easy to see that $z_{1}^{j} \rightarrow 0$ and $\liminf _{j \rightarrow \infty}\left|z_{2}^{j}\right| \geq e^{-1}$. Since $D \subset \mathbb{D}^{2}$, it follows that if $\left(\left(\hat{z}^{j}, \hat{w}^{j}\right)\right)_{j} \rightarrow 0$, then

$$
\begin{aligned}
\liminf _{j \rightarrow \infty} l_{\tilde{D}}\left(\left(z^{j}, w^{j}\right),\left(\hat{z}^{j}, \hat{w}^{j}\right)\right) & \geq \liminf _{j \rightarrow \infty} l_{D}\left(z^{j}, \hat{z}^{j}\right) \\
& \geq \liminf _{j \rightarrow \infty} l_{\mathbb{D}^{2}}\left(z^{j}, \hat{z}^{j}\right) \\
& =\liminf _{j \rightarrow \infty}\left|z_{2}^{j}\right| \geq e^{-1} .
\end{aligned}
$$


(ii) Observe that if we replace in Example $13 e^{-1}$ by any $r \in\left(e^{-1}, 1\right)$, then we have the same conclusions with $r$ instead of $e^{-1}$. In particular, since $\infty$ is not a $t$-point, it is also not a barrier point. On the other hand, using the proof of Example 4.1 (ii), it is easy to see that $\infty$ is a local barrier point for $\tilde{D}$ (with $r$ instead of $\left.e^{-1}\right)$.

Concerning tautness and hyperconvexity of Hartogs domains one has the following

Proposition 4.4. A Hartogs domain $D$ is taut (hyperconvex) if and only if $G$ is taut (hyperconvex) and $\log h$ is a continuous psh function with $\log h^{-1}(-\infty)=G \times\{0\}$.

Proof. We shall follow the proof of the analogous result in the case of bounded Hartogs domains (see [13]).

First we shall prove the implication $\Leftarrow$. Assume that $h$ has the above properties.

Suppose that $G$ is hyperconvex, i.e. it admits a negative psh exhaustion function $\varphi$. Denoting by $\tilde{\varphi}(z, w):=\varphi(z)$ the trivial extension of $\varphi$ to $D$, it is easy to see that $\max \{\tilde{\varphi}, \log h\}$ is a negative psh exhaustion function of $D$, implying that $D$ is hyperconvex.

Now assume that $G$ is taut and there exists a sequence $\left\{f_{j}\right\} \subset \mathcal{O}(\mathbb{D}, D)$ that does not diverge compactly. Then we may assume that $f_{j}^{\prime} \rightarrow f^{\prime} \in \mathcal{O}(\mathbb{D}, G)$. In particular, for any $r \in(0,1)$ we have $\cup_{j=1}^{\infty} f_{j}^{\prime}(r \mathbb{D})=: K_{r} \subset \subset D$. Since $\inf _{K_{r} \times \mathbb{S}} h>0$, it follows that $\left(f_{j}^{\prime \prime}\right)_{j}$ is uniformly bounded on $r \mathbb{D}$. By Montel's theorem one may assume that $f_{j}^{\prime \prime} \rightarrow f^{\prime \prime} \in \mathcal{O}\left(\mathbb{D}, \mathbb{C}^{m}\right)$. Set $f:=\left(f^{\prime}, f^{\prime \prime}\right)$. Using that $f^{\prime}(\mathbb{D}) \subset G$ and the continuity of $h$, we get $h(f(t)) \leq 1$ for any $t \in \mathbb{D}$. Then the maximum principle implies that either $h \circ f<1$ or $h \circ f \equiv 1$, i.e. either $f(\mathbb{D}) \in D$ or $f(\mathbb{D}) \in \partial D$.

To prove the converse implication $\Rightarrow$, note that if $D$ is pseudoconvex and hyperbolic, then $\log h$ is a psh function and $h>0$ on $G \times \mathbb{S}$. Moreover, it is clear that tautness (hyperconvexity) of $D$ implies tautness (hyperconvexity) of $G$. Assume now that $h$ is not continuous at some point $a \in D$. Then we may find a sequence $\left\{a_{j}\right\} \subset D$ and $r>1$ such that $a_{j} \rightarrow a$ for $j \rightarrow \infty$ and $(h(a))^{-1}<r \leq\left(h\left(a_{j}\right)\right)^{-1}$ for any $j$. Note that the discs $f_{j}(t):=\left(a_{j}^{\prime}, r a_{j}^{\prime \prime} t\right)$ belong to $\mathcal{O}(\mathbb{D}, D)$. On the other hand, for the limit disc $f(t):=\left(a^{\prime}, r a^{\prime \prime} t\right)$ we have $f(0) \in D$ and $f\left((h(a) r)^{-1}\right) \in \partial D$. This contradicts tautness (hyperconvexity) of $D$.

Remark. For Hartogs domains with one-dimensional fibers, the part of the statement regarding tautness is obtained in [16] (see also [14] for a more general situation). 


\section{References}

[1] M. AвATE, A characterization of hyperbolic manifolds, Proc. Amer. Math. Soc. 117 (1993), 789-793.

[2] Z. BLOCKI, The complex Monge-Ampère operator in pluripotential theory, Preprint (2004) (http://www.im.uj.edu.pl).

[3] G. T. BuZZARD and J. E. Fornaess, An embedding of $\mathbb{C}$ in $\mathbb{C}^{2}$ with hyperbolic complements, Math. Ann. 306 (1996), 539-546.

[4] B.-Y. CHEN, Bergman completeness of hyperconvex manifolds, Nagoya Math. J. 175 (2004), 165-170.

[5] B.-Y. CHEN and Z.-H. ZHANG, The Bergman metric on a Stein manifold with a bouded plurisubharminc function, Trans. Amer. Math. Soc. 354 (2002), 2997-3009.

[6] A. Eastwood, À propos des variétés hyperboliques completes, C. R. Acad. Sci. Paris 280 (1975), 1071-1075.

[7] F. FORSTNERIČ, Interpolation by holomorphic automorphisms and embeddings in $\mathbb{C}^{n}, \mathrm{~J}$. Geom. Anal. 9 (1999), 93-117.

[8] H. Gaussier, Tautness and complete hyperbolicity of domains in $\mathbb{C}^{n}$, Proc. Amer. Math. Soc. 127 (1999), 105-116.

[9] N. KeRZMAN and J.-P. RosAY, Fonctions plurisousharmoniques d'exhaustion bornées et domaines taut, Math. Ann. 257 (1981), 171-184.

[10] M. JARNICKI and P. PFLUG, Remarks on the pluricomplex Green function, Indiana Univ. Math. J. 44 (1995), 535-543.

[11] M. Jarnicki and P. Pflug, "Invariant Distances and Metrics in Complex Analysis", de Gruyter, 1993.

[12] M. JARNICKI and P. PFLUG, Invariant distances and metrics in complex analysis-revisited, Dissertationes Math. 430 (2005), 1-192.

[13] M. JARnicki, P. PFLUG and W. ZWOneK, On Bergman completeness of non-hyperconvex domains, Univ. Iagel. Acta Math. 38 (2000), 169-184.

[14] S.-H. PARK, "Tautness and Kobayashi Hyperbolicty", Ph. D. thesis, Oldenburg, 2003.

[15] N. SibONy, A class of hyperbolic manifolds, In: "Recent Developments in Several Complex Variables", J. E. Fornaess (ed.), Ann. Math. Studies 100 (1981), 347-372.

[16] Do Duc Thai and Pham VIeT Duc, On the complete hyperbolicity and the tautness of the Hartogs domains, Internat. J. Math. 11 (2000), 103-111.

[17] Do DUC ThaI and P. J. Thomas, $\mathbb{D}^{*}$-extension property without hyperbolicity, Indiana Univ. Math. J. 47 (1998), 1125-1130.

[18] Do Duc Thai and Pham Nguyen Thu Trang, Tautness of locally taut domains in complex spaces, Ann. Polon. Math. 83 (2004), 141-148.

[19] V. P. ZAHARJUTA,Extremal plurisubharmonic functions, Hilbert scales, and the isomorphism of spaces of analytic functions of several variables, Teor. Funkcii, Funkcional. Anal. i Prilozen. 19 (1974), 133-157.

[20] W. ZwoneK, Regularity properties of the Azukawa metric, J. Math. Soc. Japan 52 (2000), 899-914.

Institute of Mathematics and Informatics

Bulgarian Academy of Sciences

1113 Sofia, Bulgaria

nik@math.bas.bg

Carl von Ossietzky Universität Oldenburg

Fachbereich Mathematik

Postfach 2503

D-26111 Oldenburg, Germany

pflug@mathematik.uni-oldenburg.de 